\title{
Maximal inequalities for the best approximation op- erator and Simonenko indices
}

\author{
Sonia Acinas ${ }^{1,2, *}$ and Sergio Favier ${ }^{1,3}$ \\ ${ }^{1}$ Instituto de Matemática Aplicada San Luis, IMASL, Universidad Nacional de San \\ Luis and CONICET, Ejército de los Andes 950, D5700HHW San Luis, Argentina. \\ 2 Departamento de Matemática, Facultad de Ciencias Exactas Naturales, Universidad \\ Nacional de La Pampa, L6300CLB Santa Rosa, La Pampa, Argentina. \\ ${ }^{3}$ Departamento de Matemática, Universidad Nacional de San Luis, D5700HHW San \\ Luis, Argentina.
}

\begin{abstract}
In an abstract set up, we get strong type inequalities in $L^{p+1}$ by assuming weak or extra-weak inequalities in Orlicz spaces. For some classes of functions, the number $p$ is related to Simonenko indices. We apply the results to get strong inequalities for maximal functions associated to best $\Phi$-approximation operators in an Orlicz space $L^{\Phi}$.
\end{abstract}

Key Words: Simonenko indices, Maximal Inequalities, Best Approximation.

AMS Subject Classifications: 41A10, 41A50, 41A45.

\section{Introduction}

In this paper we denote by $\mathcal{I}$ the set of all non decreasing functions $\varphi$ defined for all real number $x>0$, such that $\varphi(x)>0$ for all $x>0, \varphi(0+)=0$ and $\lim _{x \rightarrow \infty} \varphi(x)=\infty$.

We say that a non decreasing function $\varphi: \mathbb{R}_{0}^{+} \rightarrow \mathbb{R}_{0}^{+}$satisfies the $\Delta_{2}$ condition, symbolically $\varphi \in \Delta_{2}$, if there exists a constant $\Lambda_{\varphi}>0$ such that $\varphi(2 x) \leq \Lambda_{\varphi} \varphi(x)$ for all $x \geq 0$.

Now, given $\varphi \in \mathcal{I}$, we consider $\Phi(x)=\int_{0}^{x} \varphi(t) d t$. Observe that $\Phi:[0, \infty) \rightarrow[0, \infty)$ is a convex function such that $\Phi(x)=0$ if and only if $x=0$. In the literature, a function $\Phi$ satisfying the previous conditions is known as a Young function. In addition, as $\varphi \in \mathcal{I}$ we have that $\Phi$ is increasing, $\frac{\Phi(x)}{x} \rightarrow 0$ as $x \rightarrow 0$ and $\frac{\Phi(x)}{x} \rightarrow \infty$ as $x \rightarrow \infty$. Thus, according to [6], a function $\Phi$ with this property is called an $N$-function.

If $\varphi \in \mathcal{I}$ is a right-continuous function that satisfies the $\Delta_{2}$ condition, then

$$
\frac{1}{2}(\varphi(a)+\varphi(b)) \leq \varphi(a+b) \leq \Lambda_{\varphi}(\varphi(a)+\varphi(b))
$$

*Corresponding author. Email addresses: sonia.acinas@gmail.com (S. Acinas), sfavier@unsl.edu.ar (S. Favier). 
for every $a, b \geq 0$.

Also note that the $\Delta_{2}$ condition on $\Phi$ implies

$$
\frac{x}{2 \Lambda_{\varphi}} \varphi(x) \leq \Phi(x) \leq x \varphi(x),
$$

for every $x \geq 0$.

If $\varphi \in \mathcal{I}$, we define $L^{\varphi}\left(\mathbb{R}^{n}\right)$ as the class of all Lebesgue measurable functions $f$ defined on $\mathbb{R}^{n}$ such that $\int_{\mathbb{R}^{n}} \varphi(t|f|) d x<\infty$ for some $t>0$ and where $d x$ denotes the Lebesgue measure on $\mathbb{R}^{n}$. For a convex function $\Phi, L^{\Phi}\left(\mathbb{R}^{n}\right)$ is the classic Orlicz space (see [10]). And, if $\Phi \in \Delta_{2}$ then $L^{\Phi}\left(\mathbb{R}^{n}\right)$ is the space of all measurable functions $f$ defined on $\mathbb{R}^{n}$ such that $\int_{\mathbb{R}^{n}} \Phi(|f|) d x<\infty$.

A non decreasing function $\varphi: \mathbb{R}_{0}^{+} \rightarrow \mathbb{R}_{0}^{+}$satisfies the $\nabla_{2}$ condition, denoted $\varphi \in \nabla_{2}$, if there exists a constant $\lambda_{\varphi}>2$ such that $\varphi(2 x) \geq \lambda_{\varphi} \varphi(x)$ for all $x \geq 0$.

We claim that a non decreasing function $\varphi: \mathbb{R}_{0}^{+} \rightarrow \mathbb{R}_{0}^{+}$satisfies the $\Delta^{\prime}$ condition, symbolically $\varphi \in \Delta^{\prime}$, if there exists a constant $K>0$ such that $\varphi(x y) \leq K \varphi(x) \varphi(y)$ for all $x, y \geq x_{0} \geq 0$. If $x_{0}=0$ then $\varphi$ satisfies the $\Delta^{\prime}$ condition globally (denoted $\varphi \in \Delta^{\prime}$ globally).

With the aim of comparing functions in Orlicz spaces, some partial ordering relations were treated in Chapter II of [10]. In [9] Mazzone and Zó introduce the quasi-increasing function's concept, they define the relation $\prec$ between two non negative functions and they determine some properties of the relation. Later, in [1], it is defined and thoroughly studied another relation $\prec_{N}$. Both relations are used to obtain strong type inequalities as follows.

Let $\varphi: \mathbb{R}_{0}^{+} \rightarrow \mathbb{R}_{0}^{+}$be a non decreasing function such that $\varphi(0)=0$ and satisfies a weak type inequality like

$$
\mu(\{f>a\}) \leq C_{w} \int_{\{f>a\}} \frac{\varphi(g)}{\varphi(a)} d \mu \text { for all } a>0,
$$

or an extra-weak type inequality like

$$
\mu(\{f>a\}) \leq 2 C_{w} \int_{\{f>a\}} \varphi\left(\frac{g}{a}\right) d \mu \text { for all } a>0,
$$

where $f, g: \Omega \rightarrow \mathbb{R}_{0}^{+}$are two fixed measurable functions. Then, in [9] and [1] it has considered functions $\Psi \in C^{1}([0, \infty)), \Psi(x)=\int_{0}^{x} \psi(t) d t$ and $\varphi \prec \psi$ or $\varphi \prec_{N} \psi$, which allows us to get strong type inequalities like

$$
\int_{\Omega} \Psi(f) d \mu \leq 2 C_{w} \rho \int_{\Omega} \Psi\left(\frac{2}{c} g\right) d \mu .
$$

In this paper we set $p \in \mathbb{R}$, very related to Boyd indices of $\varphi$, such that $\varphi \prec x^{p}$ or $\varphi \prec_{N} x^{p}$ in order to obtain strong inequalities like

$$
\int_{\Omega} f^{p+1} d \mu \leq \tilde{K} \int_{\Omega} g^{p+1} d \mu
$$


with $f$ and $g$ non negative, measurable functions.

In Section 2, we recall the definitions of the relations $\prec$ and $\prec_{N}$ and we enumerate some of their properties that will be useful in the searching of the real number $p$ to have (1.2) Then, we determine sufficient conditions on $p$ to have $\varphi \prec x^{p}$ or $\prec_{N} x^{p}$. From such conditions, we generate bounds for $p$ in the case that $\varphi$ is a non decreasing, differentiable function; next, we extend the results to a class of non decreasing and non differentiable functions.

In Section 3, we estimate $p$ by using a class of Boyd indices in Orlicz spaces called Simonenko indices. These indices were defined by Simonenko in [11] and they were studied in Chapter 11 of [8]. In [3], Simonenko indices are used to get Harnack's type inequalities and regularity conditions for some integral operators. In [12], relationships between Simonenko indices and other indices in Orlicz spaces are established.

In Section 4, we apply the results to a maximal function associated to best $\Phi$-approximation operators in an Orlicz space $L^{\Phi}$ and one-sided operators related to the classical Hardy-Littlewood maximal function.

\section{On relations between non negative functions}

We begin recalling a concept introduced by Mazzone and Zó in [9].

Definition 2.1. A function $\eta: \mathbb{R}^{+} \rightarrow \mathbb{R}^{+}$is quasi-increasing if and only if there exists a constant $\rho>0$ such that

$$
\frac{1}{x} \int_{0}^{x} \eta(t) d t \leq \rho \eta(x) \text { for every } x \in \mathbb{R}^{+} .
$$

Hereinafter, we will call $\rho$ the quasi increasing constant.

In [9], the relation $\prec$ between non negative functions was presented as follows.

Definition 2.2. Let $\varphi, \psi: \mathbb{R}^{+} \rightarrow \mathbb{R}^{+}$.

$\varphi \prec \psi$ if and only if $\frac{\psi}{\varphi}$ is a quasi-increasing function; that is, if and only if there exists a constant $\rho>0$ such that

$$
\frac{1}{x} \int_{0}^{x} \frac{\psi(t)}{\varphi(t)} d t \leq \rho \frac{\psi(x)}{\varphi(x)} \text { for every } x \in \mathbb{R}^{+}
$$

In Theorem 2.4 of [9], the authors employ the relation $\prec$ to get a strong type inequality like (1.1). In [1], following an analogous pattern with an extra-weak type inequality as starting point, the relation $\prec_{N}$ is defined.

Definition 2.3. Let $\varphi, \psi: \mathbb{R}^{+} \rightarrow \mathbb{R}^{+}$.

$\varphi \prec_{N} \psi$ if and only if $\left\{\psi(x) \varphi\left(\frac{\alpha}{x}\right)\right\}_{\alpha \in \mathbb{R}^{+}}$is a collection of quasi-increasing functions with 
the same quasi increasing constant; namely, if and only if there exists a constant $\rho>0$ such that

$$
\frac{1}{x} \int_{0}^{x} \psi(t) \varphi\left(\frac{\alpha}{t}\right) d t \leq \rho \psi(x) \varphi\left(\frac{\alpha}{x}\right),
$$

for every $x \in \mathbb{R}^{+}$and for every $\alpha \in \mathbb{R}^{+}$.

Remark 2.1. Note that $\prec$ is a reflexive relation while $\prec_{N}$ is not (see [1, p. 2183]).

Next, we set conditions to assure $\varphi \prec x^{p}$ or $\varphi \prec_{N} x^{p}$ for some $p \in \mathbb{R}$.

Proposition 2.1. Let $\varphi: \mathbb{R}^{+} \rightarrow \mathbb{R}^{+}$.

a) If $\frac{1}{\varphi(x)}$ is a quasi-increasing function, then $\varphi \prec x^{p}$ for every $p \geq 0$.

b) If $\left\{\varphi\left(\frac{\alpha}{x}\right)\right\}_{\alpha \in \mathbb{R}^{+}}$is a collection of quasi-increasing functions with the same quasi increasing constant, then $\varphi \prec_{N} x^{p}$ for every $p \geq 0$.

Proof. Definition 2.2 and Definition 2.3 imply that $\varphi \prec 1$ and $\varphi \prec_{N} 1$, respectively. Now, applying Proposition 3.5 of [1] with $M(x)=x^{p}$ for $p \geq 0$, we obtain $\varphi \prec x^{p}$ and $\varphi \prec_{N} x^{p}$ for every $p \geq 0$, respectively.

Proposition 2.2. Let $\varphi: \mathbb{R}^{+} \rightarrow \mathbb{R}^{+}$be a non decreasing function.

If $\varphi \in \Delta_{2}$ with $\Lambda_{\varphi}<2$, then $\varphi \prec x^{p}$ and $\varphi \prec{ }_{N} x^{p}$ for every $p \geq 0$.

Proof. We take $\psi(x)=x^{p}$ with $p \geq 0$ in Proposition 3.11 of [1].

Example 2.1. Let $\varphi(x)=\ln (\sqrt[3]{x}+1) \in \Delta_{2}$ with $\Lambda_{\varphi}<2$, then $\ln (\sqrt[3]{x}+1) \prec x^{p}$ and $\ln (\sqrt[3]{x}+$ 1) $\prec_{N} x^{p}$ for every $p \geq 0$.

It is easy to see that every non decreasing function is a quasi-increasing one (see [1, Prop. 3.4]). An immediate consequence of this fact is the following result.

Proposition 2.3. Let $p \in \mathbb{R}$.

a) If $\frac{x^{p}}{\varphi(x)}$ is a non decreasing function from $\mathbb{R}^{+}$into itself, then $\varphi \prec_{N} x^{p}$.

b) If $x^{p} \varphi\left(\frac{\alpha}{x}\right)$ is a non decreasing function from $\mathbb{R}^{+}$into itself for every $\alpha>0$, then $\varphi \prec_{N} x^{p}$.

Proposition 2.4. Let $\varphi: \mathbb{R}^{+} \rightarrow \mathbb{R}^{+}$.

Assume there exists $\tilde{\varphi}: \mathbb{R}^{+} \rightarrow \mathbb{R}^{+}$such that

$$
\tilde{\varphi}(x) \leq \varphi(x) \leq C \tilde{\varphi}(x) \text { for every } x>0 .
$$

If there exists $p \in \mathbb{R}$ such that $\tilde{\varphi} \prec x^{p}$ or $\tilde{\varphi} \prec_{N} x^{p}$, then $\varphi \prec x^{p}$ or $\varphi \prec_{N} x^{p}$.

Proof. If there exists $p \in \mathbb{R}$ such that $\tilde{\varphi} \prec x^{p}$, then there exists $\rho_{1}>0$ such that

$$
\frac{1}{x} \int_{0}^{x} \frac{t^{p}}{\tilde{\varphi}(t)} d t \leq \rho_{1} \frac{x^{p}}{\tilde{\varphi}(x)} \text { for every } x>0 .
$$


By (2.1) and (2.2), we get

$$
\frac{1}{x} \int_{0}^{x} \frac{t^{p}}{\varphi(t)} d t \leq \frac{1}{x} \int_{0}^{x} \frac{t^{p}}{\tilde{\varphi}(t)} d t \leq \rho_{1} \frac{x^{p}}{\tilde{\varphi}(x)} \leq \rho_{1} C \frac{x^{p}}{\varphi(x)},
$$

for every $x>0$. Therefore, from Definition 2.2, we have $\varphi \prec x^{p}$.

Now, if there exists $p \in \mathbb{R}$ such that $\tilde{\varphi} \prec_{N} x^{p}$, then there exists $\rho_{2}>0$ such that

$$
\frac{1}{x} \int_{0}^{x} t^{p} \tilde{\varphi}\left(\frac{\alpha}{t}\right) d t \leq \rho_{2} x^{p} \tilde{\varphi}\left(\frac{\alpha}{x}\right),
$$

for every $x>0$ and for every $\alpha>0$. By (2.1) and (2.3), we obtain

$$
\frac{1}{x} \int_{0}^{x} t^{p} \frac{1}{C} \varphi\left(\frac{\alpha}{t}\right) d t \leq \frac{1}{x} \int_{0}^{x} t^{p} \tilde{\varphi}\left(\frac{\alpha}{t}\right) d t \leq \rho_{2} x^{p} \tilde{\varphi}\left(\frac{\alpha}{x}\right) \leq \rho_{2} x^{p} \varphi\left(\frac{\alpha}{x}\right),
$$

for every $x>0$ and for every $\alpha>0$. Thus, Definition 2.3 implies that $\varphi \prec_{N} x^{p}$.

Now, given $\varphi: \mathbb{R}^{+} \rightarrow \mathbb{R}^{+}$a non decreasing function, we use Proposition 2.3 to set conditions which assure that $\frac{x^{p}}{\varphi(x)}$ and $x^{p} \varphi\left(\frac{\alpha}{x}\right)$ are non decreasing functions. We first consider the case $p>0$.

Theorem 2.1. Let $\varphi: \mathbb{R}^{+} \rightarrow \mathbb{R}^{+}$be a non decreasing, differentiable function.

If there exists $p>0$ such that $\frac{\varphi^{\prime}(x)}{\varphi(x)} \leq \frac{p}{x}$ for every $x>0$, then $\varphi \prec x^{p}$ and $\varphi \prec_{N} x^{p}$.

Proof. By elementary algebraic operations and the hypothesis on $\varphi$ and $p$, we have

$$
\left(\frac{x^{p}}{\varphi(x)}\right)^{\prime}=\frac{p x^{p-1} \varphi(x)-x^{p} \varphi^{\prime}(x)}{\varphi^{2}(x)} \geq 0 \text { for every } x>0 .
$$

Thus $\frac{x^{p}}{\varphi(x)}$ is a non decreasing function and, by Proposition 2.3, we get $\varphi \prec x^{p}$.

On the other hand, exchanging $x$ by $\frac{\alpha}{x}>0$ in the hypothesis and operating, we get

$$
\left(x^{p} \varphi\left(\frac{\alpha}{x}\right)\right)^{\prime}=p x^{p-1} \varphi\left(\frac{\alpha}{x}\right)+x^{p} \varphi^{\prime}\left(\frac{\alpha}{x}\right)\left(\frac{-\alpha}{x^{2}}\right) \geq 0,
$$

for every $x>0$ and for every $\alpha>0$.

Then, $x^{p} \varphi\left(\frac{\alpha}{x}\right)$ is a non decreasing function for every $\alpha>0$; and, Proposition 2.3 implies that $\varphi \prec_{N} x^{p}$.

Example 2.2. Let $\varphi(x)=x^{q}$ for $q>0$. As $\frac{p}{x} \geq \frac{\varphi^{\prime}(x)}{\varphi(x)}$ for every $x>0$ provided that $p \geq q>0$, then $x^{q} \prec x^{p}$ and $x^{q} \prec_{N} x^{p}$ for every $p \geq q>0$.

Example 2.3. Let $\varphi(x)=\ln (x+1)$. Since $\frac{\varphi^{\prime}(x)}{\varphi(x)} \leq \frac{p}{x}$ for every $x>0$ provided that $p \geq 1$, then $\ln (x+1) \prec x^{p}$ and $\ln (x+1) \prec_{N} x^{p}$ for every $p \geq 1$. 
Theorem 2.1 allows us to give a necessary condition in order that $\frac{x^{p}}{\varphi(x)}$ and $x^{p} \varphi\left(\frac{\alpha}{x}\right)$ are non decreasing functions.

Corollary 2.2. Let $\varphi: \mathbb{R}^{+} \rightarrow \mathbb{R}^{+}$be non a decreasing, differentiable function. If $\frac{x^{p}}{\varphi(x)}$ and $x^{p} \varphi\left(\frac{\alpha}{x}\right)$ are non decreasing with $p>0$, then $\frac{\varphi^{\prime}(x)}{\varphi(x)} \rightarrow 0$ as $x \rightarrow \infty$.

Proof. Since $\varphi: \mathbb{R}^{+} \rightarrow \mathbb{R}^{+}$is non decreasing and differentiable, then $\frac{x^{p}}{\varphi(x)}$ and $x^{p} \varphi\left(\frac{\alpha}{x}\right)$ are non decreasing with $p>0$. Next, elementary calculations as in the proof of Theorem 2.1, give us

$$
0 \leq \frac{\varphi^{\prime}(x)}{\varphi(x)} \leq \frac{p}{x} \text { for } x>0,
$$

which implies the wished result.

Example 2.4. Let $\varphi(x)=e^{x}-x-1$, then $\frac{\varphi^{\prime}(x)}{\varphi(x)} \rightarrow 1$; thus, there does not exist $p>0$ such that $\frac{x^{p}}{e^{x}-x-1}$ and $x^{p}\left(e^{\frac{\alpha}{x}}-\frac{\alpha}{x}-1\right)$ are non decreasing functions.

In a similar way to that developed in the proof of Theorem 2.1 but requesting that the derivatives of $\frac{x^{p}}{\varphi(x)}$ and $x^{p} \varphi\left(\frac{\alpha}{x}\right)$ are negative, we obtain the following result.

Proposition 2.5. Let $\varphi: \mathbb{R}^{+} \rightarrow \mathbb{R}^{+}$be a non decreasing, differentiable function. If there exists $p>0$ such that $\frac{p}{x}<\frac{\varphi^{\prime}(x)}{\varphi(x)}$ for every $x>0$, then $\frac{x^{p}}{\varphi(x)}$ and $x^{p} \varphi\left(\frac{\alpha}{x}\right)$ are decreasing.

As Lemma 3.1 of [9] establishes the background in which non increasing functions become quasi-increasing, we employ that result together with Proposition 2.5 and we get the following.

Theorem 2.3. Let $\varphi: \mathbb{R}^{+} \rightarrow \mathbb{R}^{+}$be a non decreasing, differentiable function.

If there exists $p>0$ such that $\frac{p}{x}<\frac{\varphi^{\prime}(x)}{\varphi(x)}$ for every $x>0$ and there exists $K_{p}<2^{p+1}$ such that $\frac{\varphi(x)}{\varphi\left(\frac{x}{2}\right)} \leq K_{p}$ for every $x>0$, then $\frac{x^{p}}{\varphi(x)}$ and $x^{p} \varphi\left(\frac{\alpha}{x}\right)$ are quasi-increasing, i.e. $\varphi \prec x^{p}$ and $\varphi \prec_{N} x^{p}$.

Example 2.5. Let $\varphi(x)=x^{q}$ with $q>0$, then $\frac{p}{x}<\frac{\varphi^{\prime}(x)}{\varphi(x)}$ for every $x>0$ provided that $p<q$ and $\frac{\varphi(x)}{\varphi\left(\frac{x}{2}\right)}<2^{p+1}$ for $p>q-1$.

Thus, $x^{q} \prec x^{p}$ and $x^{q} \prec_{N} x^{p}$ in the case of $\max \{q-1,0\}<p<q$.

Remark 2.2. Let $\varphi: \mathbb{R}^{+} \rightarrow \mathbb{R}^{+}$be a non decreasing function. If $\varphi$ is non differentiable, invoking Proposition 2.4, the above results for the case of differentiable functions can be employed provided that (2.1) holds.

If $p \leq 0$, it is not possible to use Theorem 2.3. However, we can establish some conditions to guarantee a relationship between $\varphi$ and $x^{p}$ by $\prec$ and $\prec_{N}$ for such values of $p$, as stated below. 
Theorem 2.4. Let $\varphi: \mathbb{R}^{+} \rightarrow \mathbb{R}^{+}$be a non decreasing function.

If there exists $p \in(-1,0]$ such that $\frac{\varphi(x)}{\varphi\left(\frac{x}{2}\right)} \leq K_{p}<2^{p+1}$ for every $x>0$, then $\varphi \prec x^{p}$ and $\varphi \prec_{N} x^{p}$.

Proof. As $\varphi$ is a non decreasing function on $\mathbb{R}^{+}$and $p \in(-1,0]$, then $\eta_{1}(x)=\frac{x^{p}}{\varphi(x)}$ and $\eta_{2}(x)=x^{p} \varphi\left(\frac{\alpha}{x}\right)$ are non increasing functions on $\mathbb{R}^{+}$. In addition, $\frac{\eta_{1}\left(\frac{x}{2}\right)}{\eta_{1}(x)} \leq K_{p}$ and $\frac{\eta_{2}\left(\frac{x}{2}\right)}{\eta_{2}(x)} \leq K_{p}$ for every $x>0$ and with $K_{p}<2^{p+1}<2$. Consequently, by Lemma 3.1 of [9] $\eta_{1}$ and $\eta_{2}$ are quasi-increasing, i.e. $\varphi \prec x^{p}$ and $\varphi \prec_{N} x^{p}$ for $p \in(-1,0]$.

Remark 2.3. In Theorem 2.4, it is required that $\varphi \in \Delta_{2}$ with $1 \leq \Lambda_{\varphi}<2$ as it has done in Proposition 3.10 of [1]. But in that case, $p$ were positive; while here, $p$ belongs to the set of real negative numbers.

Example 2.6. Let $\varphi(x)=\ln (\sqrt{x}+1)$. Then $\frac{\varphi(x)}{\varphi\left(\frac{x}{2}\right)} \leq \sqrt{2}<2^{p+1}$ provided that $p>-\frac{1}{2}$ and as it is necessary that $p \leq 0$, then $\ln (\sqrt{x}+1) \prec x^{p}$ and $\ln (\sqrt{x}+1) \prec_{N} x^{p}$ for $p \in\left(-\frac{1}{2}, 0\right]$.

\section{Simonenko indices}

Let $\Phi \in \mathcal{I}$ be an increasing, continuous function.

If $h_{\Phi}(\lambda)=\sup _{t>0} \frac{\Phi(\lambda t)}{\Phi(t)}$ with $\lambda>0$, then the numbers

$$
i(\Phi)=\lim _{\lambda \rightarrow 0^{+}} \frac{\ln h_{\Phi}(\lambda)}{\ln \lambda}=\sup _{0<\lambda<1} \frac{\ln h_{\Phi}(\lambda)}{\ln \lambda}
$$

and

$$
I(\Phi)=\lim _{\lambda \rightarrow \infty} \frac{\ln h_{\Phi}(\lambda)}{\ln \lambda}=\inf _{1<\lambda<\infty} \frac{\ln h_{\Phi}(\lambda)}{\ln \lambda},
$$

are called lower index of $\Phi$ and upper index of $\Phi$ respectively, or fundamentals indices of $\Phi$. The numbers $i(\Phi)$ and $I(\Phi)$ are also known as indices of Orlicz spaces or MatuszewskaOrlicz indices.

From (3.1) and (3.2), it is clear that $1 \leq i(\Phi) \leq I(\Phi)$. And, it is well known that $I(\Phi)<\infty$ if and only if $\Phi \in \Delta_{2}$ (see [8, Thm. 11.7]).

Moreover, if $\Phi$ and $\Psi$ are complementary Young functions, then

- $i(\Phi)>1$ if and only if $\Psi \in \Delta_{2}$;

- the pairs $i(\Psi), I(\Phi)$, and $i(\Phi), I(\Psi)$ satisfy

$$
\frac{1}{i(\Phi)}+\frac{1}{I(\Psi)}=\frac{1}{I(\Phi)}+\frac{1}{i(\Psi)}=1
$$

(see $[8$, Cor. 11.6]); 
- $\Phi \in \Delta_{2}$ is equivalent to the existence of $p_{0}, p_{1} \in[1, \infty), p_{0} \leq p_{1}$ such that

$$
C^{-1} \min \left(\lambda^{p_{0}}, \lambda^{p_{1}}\right) \Phi(t) \leq \Phi(\lambda t) \leq C \max \left(\lambda^{p_{0}}, \lambda^{p_{1}}\right) \Phi(t),
$$

for some constant $C>0$ and for every $\lambda, t \geq 0$; and, $\sup p_{0}=i(\Phi)$ and $\inf p_{1}=I(\Phi)$ ( [4]);

- from (3.3) other formulae for $i(\Phi)$ and $I(\Phi)$ are obtained, i.e.

$$
\begin{aligned}
& i(\Phi)=\sup \left\{p: \inf _{\substack{u>0 \\
\lambda \geq 1}} \lambda^{-p} \frac{\Phi(\lambda u)}{\Phi(u)}>0\right\}, \\
& I(\Phi)=\inf \left\{p: \sup _{\substack{u>0 \\
\lambda \geq 1}} \lambda^{-p} \frac{\Phi(\lambda u)}{\Phi(u)}<\infty\right\}
\end{aligned}
$$

(c.f. $[8$, Thm. 11.13]).

In [11] and [8] other indices, related to (3.1) and (3.2), are introduced.

Definition 3.1. Let $\Phi \in \mathcal{I}$ be an increasing, differentiable function and assume that $\Phi=$ $\int_{0}^{x} \varphi(t) d t$.

If there exist $p, q \in \mathbb{R}$ such that

$$
p \Phi(t) \leq x \varphi(x) \leq q \Phi(x) \text { for every } x \in \mathbb{R},
$$

then the best $p$ and $q$ that verify (3.4) are called Simonenko indices and they satisfy

$$
p(\Phi)=\inf _{x>0} \frac{x \varphi(x)}{\Phi(x)} \text { and } q(\Phi)=\sup _{x>0} \frac{x \varphi(x)}{\Phi(x)} .
$$

Remark 3.1. The relationship between Simonenko indices and indices of Orlicz spaces is given by the following inequality

$$
p(\Phi) \leq i(\Phi) \leq I(\Phi) \leq q(\Phi),
$$

that was proved in Theorem 11.11 of [8].

If $\Phi$ and $\Psi$ belong to $C^{1}$ and they are complementary $N$-functions, then

- $\Phi \in \Delta_{2}$ if and only if $q(\Phi)<\infty$ (c.f. [10, Cor. 4, pp. 22-23]);

- $\Psi \in \Delta_{2}$ if and only if $1<p(\Phi)$ (c.f [10, Cor. 4, pp. 22-23]);

- $\Phi \in \Delta_{2} \cap \nabla_{2}$ if and only if $1<p(\Phi) \leq q(\Phi)<\infty$. 
- As in the case of indices of Orlicz spaces, Simonenko indices of complementary, $N$-functions behave as the conjugate exponents of the power functions, i.e.

$$
\frac{1}{p(\Phi)}+\frac{1}{q(\Psi)}=1=\frac{1}{p(\Psi)}+\frac{1}{q(\Phi)}
$$

( [10, Cor. 6, p. 27]).

Now, we get power functions related to some function $\Phi$ by $\prec$ or $\prec_{N}$ employing Simonenko indices.

Theorem 3.1. Let $\Phi \in C^{1}$ be an $N$-function that satisfies the $\Delta_{2}$ condition. If $q(\Phi)$ is the upper Simonenko index, then

1) $\Phi \prec x^{q(\Phi)}$ and $\Phi \prec_{N} x^{q(\Phi)}$;

2) $\Phi \prec x^{q}$ and $\Phi \prec_{N} x^{q}$ for every $q \geq q(\Phi)$.

Proof. 1) Since $\Phi$ is a differentiable, $N$-function that satisfies the $\Delta_{2}$ condition, there exists $q(\Phi)<\infty$ such that $\frac{\varphi(x)}{\Phi(x)} \leq \frac{q(\Phi)}{x}$ for every $x>0$. Next, by Theorem 2.1 we have $\Phi \prec x^{q(\Phi)}$ and $\Phi \prec_{N} x^{q}(\Phi)$.

2) By the definition of $q(\Phi)$, we have $\frac{\varphi(x)}{\Phi(x)} \leq \frac{q}{t}$ for every $q \in[q(\Phi), \infty)$ and for every $x>0$, then $\Phi \prec x^{q}$ and $\Phi \prec_{N} x^{q}$ for every $q \geq q(\Phi)$.

Remark 3.2. If $\Phi \in \Delta_{2}$, then $1 \leq \frac{x \varphi(x)}{\Phi(x)} \leq 2 \Lambda_{\varphi}$ for every $x>0$ and therefore $1 \leq p(\Phi) \leq q(\Phi)<\infty$. If, in addition, $\Phi \in \nabla_{2}$, then Theorem 3.1 is satisfied with $q(\Phi) \in(1, \infty)$.

Example 3.1. c.f. [8].

If $\Phi(x)=x^{p}\left(1+\frac{1}{\sqrt{5}} \sin (p \ln x)\right)$ for $x \in(0, \infty)$ and with $p \geq 6$, then $\Phi$ is an $N$-function such that $\Delta_{2} \cap \nabla_{2}$ and $p(\Phi)=\frac{1}{2} p$ y $q(\Phi)=\frac{3}{2} p$. Now, by Theorem 3.1, $\Phi \prec x^{\frac{3}{2} p}$ and $\Phi \prec_{N} x^{\frac{3}{2} p}$ with $p \geq 6$.

Theorem 3.2. Let $\Phi \in C^{1}$ be an $N$-function that satisfies the $\Delta_{2}$ condition and let $p(\Phi)$ be the lower Simonenko index.

1) If there exists $K_{p(\Phi)}<2^{p(\Phi)+1}$ such that $\frac{\Phi(x)}{\Phi\left(\frac{x}{2}\right)} \leq K_{p(\Phi)}$ for every $x>0$, then $\Phi \prec x^{p(\Phi)}$ and $\Phi \prec_{N} x^{p(\Phi)}$.

2) If $0<p \leq p(\Phi)$ and there exists $K_{p}<2^{p+1}$ such that $\frac{\Phi(x)}{\Phi\left(\frac{x}{2}\right)} \leq K_{p}$ for every $x>0$, then $\Phi \prec x^{p}$ and $\Phi \prec_{N} x^{p}$.

Proof. 1) If $\Phi$ is a differentiable, $N$-function such that $\Phi \in \Delta_{2}$, then there exists $0<p(\Phi)<\infty$ such that $\frac{\varphi(x)}{\Phi(x)} \geq \frac{p(\Phi)}{x}$ for every $x>0$. If there also exists $K_{p(\Phi)}<2^{p(\Phi)+1}$ such that $\frac{\Phi(x)}{\Phi\left(\frac{x}{2}\right)} \leq$ $K_{p(\Phi)}$ for every $x>0$ then, by Theorem $2.3, \Phi \prec x^{p(\Phi)}$ and $\Phi \prec_{N} x^{p(\Phi)}$.

2) From the definition of $p(\Phi)$, we have $\frac{\varphi(x)}{\Phi(x)} \geq \frac{p}{x}$ for every $p \in(0, p(\Phi)]$ and for every $t>0$; and, as there exists $K_{p}<2^{p+1}$ such that $\frac{\Phi(x)}{\Phi\left(\frac{x}{2}\right)} \leq K_{p}$ for every $x>0$, then $p \leq p(\Phi)$ and therefore $\Phi \prec x^{p}$ and $\Phi \prec_{N} x^{p}$. 
Remark 3.3. If it is also assumed that $\Phi \in \nabla_{2}$, then Theorem 3.2 holds for $p(\Phi) \in(1, \infty)$.

Example 3.2. If $\Phi(x)=x^{\alpha}$ with $\alpha \geq 1$, then $p(\Phi)=q(\Phi)=\alpha$. In addition, if $\Phi \in \Delta_{2}$ with $\Lambda_{\Phi}<2^{\alpha+1}$, then $\Phi \prec \Phi$ and $\Phi \prec{ }_{N} \Phi$.

Let $0<p \leq \alpha$ such that $\alpha-1<p$, then $\Phi \in \Delta_{2}$ with $\Lambda_{\Phi}<2^{p+1}$ and therefore $x^{\alpha} \prec x^{p}$ and $x^{\alpha} \prec_{N} x^{p}$ for $0<p \leq \alpha$.

\section{$4 \quad$ Main Result}

Let $(\Omega, \mathcal{A}, \mu)$ be a finite measure space.

A subset $\mathcal{L} \subset \mathcal{A}$ is a $\sigma$-lattice if and only if $\varnothing, \Omega \in \mathcal{L}$ and $\mathcal{L}$ is closed under countable unions and intersections.

A function $f: \Omega \rightarrow \mathbb{R}$ is said to be $\mathcal{L}$-measurable if $\{f>a\} \in \mathcal{L}$ for all $a \in \mathbb{R}$.

We denote by $L^{\Phi}(\mathcal{L})$ the set of all $\mathcal{L}$-measurable functions in $L^{\Phi}(\Omega)$.

Definition 4.1. A function $g \in L^{\Phi}(\mathcal{L})$ is called a best $\Phi$-approximation to $f \in L^{\Phi}(\Omega)$ if and only if $\int_{\Omega} \Phi(f-g) d \mu=\min _{h \in L^{\Phi}(\mathcal{L})} \int_{\Omega} \Phi(f-h) d \mu$.

Let $\mu(f, \mathcal{L})$ be the set of all best $\Phi$-approximations to $f \in L^{\Phi}(\Omega)$. It is well known that for each $f \in L^{\Phi}(\Omega)$, the set $\mu(f, \mathcal{L})$ is not empty (see [7]).

Suppose that $\mathcal{L}_{n}$ is an increasing sequence of $\sigma$-lattices, i.e. $\mathcal{L}_{n} \subset \mathcal{L}_{n+1}$ for every $n \in \mathbb{N}$. Let $f$ be a non negative function of $L^{\Phi}(\Omega)$ and let $f_{n}$ be any selection of functions of $\mu\left(f, \mathcal{L}_{n}\right)$.

In [9] it is defined the maximal function $f^{*}=\sup _{n} f_{n}$ and the authors obtain strong type inequalities in some $L^{\Psi}$ spaces.

Next, we will get strong type inequalities for $f^{*}$ in some $L^{p}$ spaces employing the results of the previous sections where Simonenko indices are involved.

Theorem 4.1. Let $\Phi \in C^{2}([0, \infty))$ and let $\varphi$ be its derivative. Assume that $\varphi$ is an N-function such that $\varphi(0)=0$ and $\varphi \in \Delta^{\prime}$ globally.

If $q(\varphi)$ is the upper Simonenko index, then

$$
\int_{\Omega}\left(f^{*}\right)^{q(\varphi)+1} d \mu \leq 2 K C_{q}\left(\frac{2}{c}\right)^{q(\varphi)+1} \int_{\Omega} f^{q(\varphi)+1} d \mu,
$$

for some $c>0$ and with $K C_{q}$ independent of $f$.

If $p(\varphi)$ is the lower Simonenko index and there exists $K_{p(\varphi)}<2^{p(\varphi)+1}$ such that $\frac{\varphi(x)}{\varphi\left(\frac{x}{2}\right)} \leq K_{p(\varphi)}$ for every $x>0$, then

$$
\int_{\Omega}\left(f^{*}\right)^{p(\varphi)+1} d \mu \leq 2 K C_{q}\left(\frac{2}{c}\right)^{p(\varphi)+1} \int_{\Omega} f^{p(\varphi)+1} d \mu,
$$

for some $c>0$ and with $K C_{q}$ independent of $f$. 
Proof. As $\varphi(0)=0$ and $\varphi \in \Delta^{\prime}$ globally, by [AF12, Thm. 4.5] we have

$$
\mu\left(\left\{f^{*}>a\right\}\right) \leq K \int_{\left\{f^{*}>a\right\}} \varphi\left(\frac{f}{a}\right) d \mu \text { for every } a>0,
$$

with $K$ depending on $\Lambda_{\Phi}$.

Since $\varphi \in C^{1}([0, \infty))$, it is also true that $\varphi(0+)=0$. Now, from [1, Lemma 2.1], there exists $c>0$ such that

$$
\mu\left(\left\{f^{*}>a\right\}\right) \leq 2 K \int_{\{f>c a\}} \varphi\left(\frac{f}{a}\right) d \mu \text { for every } a>0 .
$$

On the other hand, by Theorem 3.1 we have $\varphi \prec_{N} x^{q(\varphi)}$; and, from Theorem 3.2, we get $\varphi \prec_{N} x^{p(\varphi)}$ provided that there exists $K_{p(\varphi)}<2^{p(\varphi)+1}$ such that $\frac{\varphi(x)}{\varphi\left(\frac{x}{2}\right)} \leq K_{p(\varphi)}$ for every $x>0$. Finally, by (4.4) and [1, Thm. 3.17], we obtain (4.1) and (4.2).

Example 4.1. Let $\varphi(x)=x^{\alpha}\left(1+\frac{1}{\sqrt{5}} \sin (\alpha \ln x)\right)$ for $x \in(0, \infty)$ and $\alpha \geq 6$, then $\varphi(0+)=0$ and $\varphi \in \Delta^{\prime}$. The characteristics of $\varphi$ guarantee that (4.3) and (4.4) are satisfied.

On the other hand, by Example 3.1 we have $\varphi \prec x^{\frac{3}{2} \alpha}$ and $\varphi \prec_{N} x^{\frac{3}{2} \alpha}$ with $\alpha \geq 6$ being $p(\varphi)=\frac{1}{2} \alpha$ and $q(\varphi)=\frac{3}{2} \alpha$ the lower and upper Simonenko indices of $\varphi$, respectively.

As a consequence,

$$
\int_{\Omega}\left(f^{*}\right)^{\frac{3}{2} \alpha+1} d \mu \leq 2 K C_{q}\left(\frac{2}{c}\right)^{\frac{3}{2} \alpha+1} \int_{\Omega} f^{\frac{3}{3} \alpha+1} d \mu,
$$

for $\alpha \geq 6$ and where $f$ is a non negative function in $L^{\Phi}(\Omega)$.

Remark 4.1. If $\varphi$ is not an $N$-function, but $\varphi$ is the right continuous derivative of a Young function $\Phi, \varphi(0)=\varphi(0+)=0$, and $\varphi \in \Delta^{\prime}$, we have that Proposition 2.1 or Proposition 2.2, provides some $p>-1$ such that $\varphi \prec_{N} x^{p}$, depending on the properties of $\varphi$. Then,

$$
\int_{\Omega}\left(f^{*}\right)^{p+1} d \mu \leq 2 K C_{q}\left(\frac{2}{c}\right)^{p+1} \int_{\Omega} f^{p+1} d \mu,
$$

for every $f \in L^{\Phi}(\Omega)$.

In $[9, \mathrm{Thm} .1 .1]$, it is proved that

$$
\mu\left(\left\{f^{*}>a\right\}\right) \leq \frac{C}{\varphi_{+}(a)} \int_{\left\{f^{*}>a\right\}} \varphi_{+}(f) d \mu \text { for every } a>0,
$$

with $C$ depending only on $\Lambda_{\Phi}$ and where $\varphi_{+}$is the right derivative of the Young function $\Phi, \varphi_{+} \in \Delta_{2}$ and $\varphi_{+}(0)=0$. Then, by a similar procedure to that developed in the proof of Theorem 4.1, strong type inequalities for $f^{*}$ in some $L^{p}$ spaces can be obtained by means of the relation $\prec$ instead of $\prec_{N}$ ( [9, Cor. 2.4]). However, we point out that it is not possible to carry out such a procedure assuming only $\varphi_{+}(0)=\varphi_{+}(0+)=0$. In fact, it is necessary to ask an additional condition on $\varphi_{+}$, which is the existence of a constant $r \in(0,1)$ such that $\varphi_{+}(r x) \leq \frac{1}{2} \varphi_{+}(x)$ for every $x>0$ (c.f. [9, Lemma 2.2]). 
Example 4.2. Let $\varphi(x)=\ln (x+1)$. Then, $\varphi(0)=\varphi(0+)=0$ and $\varphi \in \Delta^{\prime}$ globally. Then (4.5) and (4.3) are satisfied and there also exists $c>0$ such that (4.4) holds.

In Example 2.3 we have seen that $\ln (x+1) \prec_{N} x^{p}$ for every $p>1$, then we obtain

$$
\int_{\Omega}\left(f^{*}\right)^{p+1} d \mu \leq 2 K C_{q}\left(\frac{2}{c}\right)^{p+1} \int_{\Omega} f^{p+1} d \mu
$$

for every $f \in L^{\Phi}(\Omega)$ and with $p>-1$ such that $\varphi_{+} \prec_{N} x^{p}$.

Now, as there does not exist $r \in(0,1)$ such that $\ln (r x+1) \leq \frac{1}{2} \ln (x+1)$ for every $x>0$, we cannot get (4.6) having (4.5) as a starting point, because we cannot apply [9, Lemma 2.2], although (4.5) is valid and $\ln (x+1) \prec x^{p}$ for every $p>1$.

\subsection{Final remarks on some one-sided operators}

Proposition 5.1 of [1] allows us to obtain valid weak type inequalities on proper subsets of $\mathbb{R}$ for the classical Hardy-Littlewood maximal function $M$ and the one-sided HardyLittlewood maximal functions $M^{ \pm}$, from weak type inequalities that hold true on the whole $\mathbb{R}^{n}$. In Theorem 2, Theorem 3 and Theorem 5 of [2] conditions on $\varphi \in \mathcal{I}$ to have weak type inequalities for $M^{ \pm}$on the whole $\mathbb{R}$ were established; next, by Proposition 5.1 of [1], we obtain

$$
\left|\left\{x \in \mathbb{R}:\left|M^{ \pm} f(x)\right|>\lambda\right\}\right| \leq C \int_{\left\{x:|f(x)|>\frac{\lambda}{2}\right\}} \varphi\left(\frac{2 C f(x)}{\lambda}\right) d x
$$

for all $\lambda>0$ and where the constant $C$ is independent of the function $f$. Then, (1.1) holds with $f=M^{ \pm}$and for values of $p$ that depend on the characteristics of $\varphi$, that is,

$$
\int_{\mathbb{R}}\left(M^{ \pm} f\right)^{p+1} d x \leq \tilde{K} \int_{\mathbb{R}} f^{p+1} d x
$$

for every non negative function $f \in L_{l o c}^{1}(\mathbb{R})$, with $p>-1$ such that $\varphi \prec_{N} x^{p}$ and where $\tilde{K}$ is independent of $f$.

Remark 4.2. In Theorem 7 of [2] strong type inequalities for $M^{ \pm}$are characterized, as it has done in [5] for the Hardy-Littlewood maximal function $M$.

One-sided maximal operators $\mathcal{M}^{ \pm}$, associated to one-sided best approximation by constants, were defined and studied in [2, pp. 155-160]. The relationship between $\mathcal{M}^{ \pm}$ and $M^{ \pm}$is established in Lemma 1 of [2]. Using this relationship, strong type inequalities for the one-sided maximal operators $\mathcal{M}^{ \pm}$like (1.1) can be obtained from (4.7).

A similar situation occurs with the operators $M_{p}^{ \pm}$(see [2, pp. 160-161]) because they were defined from the one-sided Hardy-Littlewood maximal functions $M^{ \pm}$. 


\section{Funding}

This paper was supported by Consejo Nacional de Investigaciones Científicas y Técnicas (CONICET) and Universidad Nacional de San Luis (UNSL) with grants PIP 11220110100033CO and PROICO 317902, respectively.

\section{Acknowledgments}

The authors would like to thank the referees for their valuable comments and suggestions.

\section{References}

[1] S. Acinas and S. Favier, Maximal Inequalities in Orlicz spaces, Int. J. Math. Anal. (Ruse), 6:41-44 (2012), 2179-2198.

[2] S. Acinas and S. Favier, Inequalities for one-sided operators in Orlicz spaces, Actas del XII Congreso Dr. Antonio A. R. Monteiro, 12 (2014), 149-161.

[3] A. Fiorenza and M. Krbec: Indices of Orlicz spaces and some applications, Comment. Math. Univ. Carolin., 38:3 (1997), 433-451.

[4] J. Gustavsson and J. Peetre, Interpolation of Orlicz spaces, Studia Mathematica, 60:1 (1977), 33-59.

[5] V. Kokilashvili, M. Krbec, Weighted Inequalities in Lorentz and Orlicz Spaces, World Scientific, Singapore, 1991.

[6] M. A. Krasnosel'skii and Ya. B. Rutickii, Convex Functions and Orlicz Spaces, P. Noordhoff Ltd., Groningen, The Netherlands, 1961.

[7] D. Landers, L. Rogge, Best approximants in $L_{\Phi}$-spaces, Z. Wahrsch. Verw. Gabiete, 51 (1980), 215-237.

[8] L. Maligranda, Orlicz Spaces and Interpolation, Seminarios de Matemática, Campinas SP., Brasil, 1989.

[9] F.D. Mazzone, F. Zó, On Maximal Inequalities Arising in Best Approximation, J. Inequal. Pure and Appl. Math., 10:2 (2009), Article 58, 10 pp.

[10] M. Rao, Z. Ren, Theory of Orlicz Spaces, Marcel Dekker Inc., New York, 1991.

[11] I. B. Simonenko, Interpolation and extrapolation of linear operators in Orlicz spaces, Dokl. Akad. Nauk; SSSR, 151 (1963), 1288-1291.

[12] Yakiang Yan, Relationship between Matuszewska-Orlicz, Semenov and Simonenko indices of $\Phi$ - functions, Publ. Inst. Math. (Beograd) (N.S.), 73(87), 2003, 139-147. 